\title{
DEPOIS DA EDUCAÇÃO A DISTÂNCIA: TECNOLOGIA, EDUCAÇÃO E FORMAÇÃO NA ITÁLIA $(1995-2008)^{*}$
}

\author{
Pier Cesare Rivoltella ${ }^{* *}$
}

\begin{abstract}
RESUMO: $\mathrm{O}$ artigo pretende fornecer um quadro-síntese da educação a distância na Itália. A análise feita trabalha sobre um duplo nível de reconstrução do seu desenvolvimento no país, das primeiras experiências nos anos cinqüenta até hoje, e da elaboração teórica que foi produzida pela literatura especializada. São considerados três âmbitos: a escola, a universidade e a empresa. Em cada um são feitas referências às experiências de maior relevo desenvolvidas nos últimos anos. A hipótese teórica que fundamenta esta contribuição e que se expressa plenamente na conclusão é que os instrumentos da Web 2.0 estão predispondo a uma mudança de paradigma capaz de dar novo impulso a um movimento e a um mercado que, nos últimos anos, está estagnado sobre os modelos e as experiências-padrão.
\end{abstract}

Palavras-chave: E-learning. Educação a distância. Escola. Universidade. Web 2.0

\section{AFTER DisTANCE EDUCATION: \\ TECHNOLOGIES, EDUCATION AND TRAINING IN ITALY (1995-2008)}

ABSTRACT: The aim of this article is to give a brief framework of distance education in Italy. Doing that it works on two levels: the first one is the history of distance education in the Country, from first experiences in the 50thies 'til nowadays; the second one is the theoric debate among the scholars about the key-concepts of this area. Three main items are considered: the School, the University,

\footnotetext{
Tradução de Monica Fantin, com revisão técnica do autor.

** Diretor do Centro di Ricerca sull'Educazione ai Media, all'Informazione e alla Tecnologia (CREMIT) e professor titular de Tecnologie dell'Istruzione e dell'Apprendimento da Università Cattolica del S. Cuore (UCSC), Milano (Itália).E-mail: piercesare.rivoltella@unicatt.it
} 
and the Enterprise. Of anyone are analyzed the best practices. The theoric hypothesis, clarified in the conclusions of this article, is that Web 2.0 tools are deeply transforming the situation: they'll probably be able to give new dynamism to a movement and to a market that in the last times seemed too fixed on standard models and experiences.

Key words: E-learning. Distance education. School. University. Web 2.0.

\section{Quadro conceitual e evolutivo: entre práticas e saberes}

literatura da área formativa e tecnológica na língua italiana recorre a expressóes muito diversificadas para definir, aparentemente, o mesmo fenômeno: educação a distância, formação a distância, formação on-line, formação em rede, ensino a distância, teledidática, teleformação. O elemento unificador de todas estas formulações é seguramente a distância, ou seja, aquele que em outros termos é definido como o "desligamento em relação ao espaço" (Rivoltella, 2003): em essência, graças a este modelo de formação, o compartilhamento de lugar não representa mais a condição sem a qual não é possível haver comunicação formativa. A formação chega também a quem não está presente, torna-se acessível também para o trabalhador e, de qualquer modo, para todos aqueles que por diversos motivos não tiveram a possibilidade de ir às aulas. Além disso, os métodos e as técnicas por meio das quais o compartilhamento de lugar é abolido podem ser muito diferentes, tão diferentes que configuram verdadeiras e diferentes "idades" da educação a distância. Trentin (1999) mostra isso muito bem quando, retomando a modelagem de Nipper (1989), formaliza na "história da educação a distância" três fases, três épocas que ele denomina educação a distância de primeira, segunda e terceira geração. Adotamos estas fases para descrever sinteticamente a evolução das práxis e da reflexão italiana em matéria de educação a distância (EaD).

A EaD de primeira geração (anos de 1950/60) é ligada à tecnologia do livro e ao desenvolvimento do sistema postal. As intervenções formativas atribuídas a esta primeira geração servem-se de materiais impressos (livros, apostilas), são baseadas na correspondência (a instituição de formação envia o material à casa do estudante e o estudante envia seus trabalhos e testes para a avaliação à instituição) e não se prevê 
a interação entre professor e estudante, senão em raros encontros presenciais.

Quando o material impresso é integrado por outros suportes multimediais (como a gravação em áudio, a filmagem televisiva e, mais recentemente, o material informático - das primeiras estruturas de Courseware ao CD-ROM), passa-se à EaD de segunda geração (anos de $1960 / 80)$. O sistema formativo, como na primeira geração, continua a ser caracterizado substancialmente para a auto-instrução; a interação professor-aluno ainda é escassa, mesmo que a possibilidade de ligação telefônica, do fax e mais recentemente do correio eletrônico permita ativar uma interação com maior freqüência e eficácia.

Quando a rede internet começa a desenvolver sistemas de comunicação mediada pelo computador ( $\mathrm{CMC}$ ), facilitando a interação síncrona (chat) e assíncrona (fórum) do professor com os estudantes e dos estudantes entre si, ocorre a passagem para a EaD de terceira geração (da metade dos anos de 1990 em diante). Na Itália esta transição acontece depois de 1995 por meio de algumas experimentaçôes e vai consolidando-se após 1998, ano "da virada" relacionada à penetração social da internet. ${ }^{1}$ E neste período que a expressão "educação a distância de terceira geração" começa a ser substituída por outras: fala-se de "aprendizagem ciberespacial" (Gallino, 1995), de "formação em rede" (Rivoltella, 1999) ou de "formação on-line" (Calvani \& Rotta, 2000). A mudança lingüística testemunha a superação do paradigma da distância: a formação que é possível desenvolver por meio do novo aporte do computador e das redes telemáticas não encontra mais o seu plus na possibilidade de suprimir a distância; além da abolição da distância, o que caracteriza este modo de fazer formação é a possibilidade de fazer com que os sujeitos possam colaborar e cooperar, a co-construção do conhecimento, e a marcada interação entre os sujeitos da formação (Calvani, 2005; Ferrari, 2006).

Junto à importação e adoção de modelos construtivistas desenvolvidos no contexto anglo-saxão (demonstrada em muitos trabalhos, entre os quais: Calvani \& Rotta, 1999; Trentin, 2001; Lodrini, 2002), esta mudança semântica favorece o alinhamento da pesquisa e do mercado da formação a respeito das práticas internacionais, sobretudo anglo-saxôes, e corresponde à afirmação social do termo e-learning para definir o conjunto de práticas educativas e formativas que se podem 
construir através da predisposição dos ambientes telemáticos. A literatura mais recente (posterior a 2002) é testemunha desta reconfiguração (Ghislandi, 2002; Di Nicola \& Comunello, 2003; Scurati, 2004; Ranieri, 2005; Galliani \& Costa, 2005; Ferri, 2005; Crispiani \& Rossi, 2006), além de um fato muito curioso que tentaremos mostrar e sobre o qual tornaremos mais adiante: quanto mais o e-learning está presente nos livros e nos artigos, mais se retira das práticas formativas e dos investimentos das empresas para configurar uma situação - a atual - que, segundo alguns pontos de vista, podem-se definir como crise ou normalização. Em que termos, veremos mais adiante, avaliando também a possibilidade de sair desta fase graças ao aporte da nova revolução tecnológica produzida pelo advento da Web 2.0.

\section{Figura 1}

Da EaD ao e-learning na Itália (1950-2000)

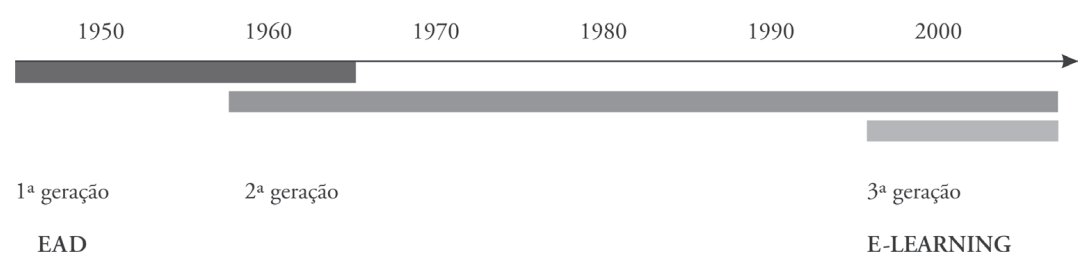

Da primeira a segunda geração de EaD: uma história que continua

A afirmação da educação a distância na Itália é ligada à situação particular do pós-guerra. O país saía destruído de vinte anos de ditadura, trazia pesadíssimos sinais dos bombardeios, tinha a necessidade de reconstruir-se tanto urbanisticamente como na sua capacidade produtiva. A carência mais evidente que logo se apresenta é a necessidade de competência profissional: faltam trabalhadores especializados e aqueles que poderiam estar interessados em uma formação neste sentido já estão ocupados e não podem deixar o seu trabalho para voltar a estudar. Além disso, os estudos custam e repercutiriam consideravelmente sobre os salários, em geral, baixos. Outra questão crucial diz respeito ao analfabetismo que, em muitas áreas do país, sobretudo na parte meridional, chegava a $24 \%$. 
É neste contexto que se devem colocar as duas primeiras experiências significativas de EaD italianas: a primeira é constituída pela fundação da Scuola Radio Elettra di Torino e a segunda pelo início das transmissōes de Telescuola, pela RAI (Radiotelevisione Italiana, Rede Pública de Rádio e Televisão Italiana).

A Scuola Radio Elettra di Torino foi fundada em 1951, em Torino, pelo refugiado polonês Tomas Carter Paszkowski e pelo torinense Vittorio Veglia. Nascida para ensinar a consertar, por correspondência, aparelhos radiofônicos (nos últimos anos do boom econômico, o rádio era um verdadeiro meio de comunicação de massa na Itália), a escola torna-se em poucos anos a maior instituição por correspondência não só da Itália, mas de diversos países europeus, onde rapidamente começa a abrir suas sedes. A metodologia didática é caracterizada pela autoinstrução: a escola envia o material de estudo impresso à casa do estudante inscrito; o estudante lê, estuda e reenvia os testes à escola para fazer a avaliação. Desde 1951, quase um milhão de pessoas são formadas seguindo estes cursos, pelo menos até o surgimento da televisão e os progressos do sistema de instrução não começarem a decretar o lento declínio da escola.

É justamente a televisão que protagoniza outro case history que mostraremos. A Telescuola é um programa televisivo da RAI desenvolvido em colaboração com o Ministério da Instrução Pública para permitir que os estudantes residentes em zonas do país em que faltam escolas completem o segundo ciclo de ensino obrigatório. As transmissōes começam em 25 de novembro de 1958 e continuam até 1966. A partir de 15 de novembro de 1960, começa a ser difundida outra transmissão televisiva, Non è mai troppo tardi (Nunca é tarde demais), dirigida aos adultos analfabetos com o objetivo preciso de ensiná-los a ler e escrever (e de fato, com os 484 programas no ar até 1968, cerca de um milhão de italianos seriam alfabetizados desta forma). O idealizador e apresentador da transmissão é Alberto Manzi, pesquisador universitário, professor de escola, ligado à cultura brasileira e às técnicas ativas de educação de adultos propostas por Paulo Freire (Novara, 2002). Na Itália, com justo título, Manzi é considerado um dos pais da EaD: habilíssimo no desenho, no seu programa explora a televisão (da qual demonstra conhecer a linguagem), as imagens e as técnicas de animação com grande habilidade para dar vida a um formato ainda hoje atualíssimo. 
Os cursos por correspondência e o modelo da formação televisiva sobrevivem até os dias de hoje, "atualizados" com a integração das técnicas de e-learning. A Scuola Radio Elettra (disponível na internet: http://www.scuolaradioelettra.it/it/sre) possui um portal, realiza as suas inscrições on-line, ampliou a sua "missão" também ao suporte formativo para recuperação das obrigaçóes escolares, mas perdeu de vista os seus objetivos originais. A formação tornou-se cara, tanto que no E-Bay surgiu um verdadeiro mercado para a compra e venda de materiais e instrumentos usados por estudantes da Radio Elettra.

A Telescuola terminou as suas transmissões há mais de trinta anos, mas o modelo de formação televisiva permanece presente, considerando que o maior consórcio italiano para a formação universitária a distância, NETTUNO (disponível na internet: http://www.consorzionettuno.it/ nettuno/index.htm), usufrui de dois canais por satélites disponíveis pela RAI para transmitir as suas videoaulas.

\section{E-learning: âmbitos e experiências}

Os limites das experiências a que fizemos referências nos parágrafos precedentes são evidentes. Além do fato de que permitem substituir outras oportunidades de formação que, por diversos motivos, não são possíveis (formação substitutiva), os sistemas de primeira e segunda geração não garantem uma adequada personalização da intervenção formativa e são de natureza fortemente transmissiva, não permitindo à formação abrir-se ao trabalho colaborativo nem às atividades de projetos coletivos. Por estes motivos, entre o final dos anos de 1990 e o ano 2000, são rapidamente substituídas pelos sistemas de e-learning. Vejamos as experiências mais interessantes a esse respeito na escola, na universidade e na empresa.

\section{Escola}

$\mathrm{Na}$ escola, o e-learning encontrou nos últimos trinta anos um grande laboratório de desenvolvimento no âmbito da formação em serviço dos professores e do pessoal administrativo (diretores administrativos, funcionários de secretaria, técnicos de laboratório). Isso remonta a 2001, quando o Ministério da Instrução Pública decide iniciar um 
plano de transformação da formação institucional dos mais de 900 mil trabalhadores da escola. O plano passa pela requalificação da Biblioteca de Documentação Pedagógica (BDP) de Firenze, que foi criada em 1925 como Mostra Nacional da Didática e depois transformada em Museu Nacional da Escola (1938) e no Centro Didático de Estudos e Inovação nos anos de 1950. A Biblioteca é transformada no Istituto Nazionale per la Documentazione dell'Insegnamento de della Ricerca Educativa - Instituto Nacional para a Documentação do Ensino e da Pesquisa Educativa (INDIRE), disponível na internet: http://www.indire.it/\#) - com a função específica de ocupar-se da formação profissional de professores e funcionários da escola. A Agência desenvolveu um ambiente on-line, Puntoedu (disponível na internet: http://puntoedu.indire.it/ corsi), no interior do qual são hospedados ano a ano os programas de formação e o repositório de todos os conteúdos didáticos; estes conteúdos estão disponíveis aos professores em serviço inscritos na comunidade For, independentemente do fato de estarem seguindo um programa de formação. $\mathrm{O}$ modelo de formação adotado é do tipo blended: sobre todo o território nacional são constituídas turmas formadas por 25 professores; cada turma é coordenada por um professor-tutor; a formação acontece em cada turma através de alguns encontros presenciais, momentos de interação em aula virtual (utilizando como ambiente o Macromedia Breeze) e atividades individuais e em pequenos grupos voltadas para a realização de exercícios.

Neste modelo de formação, um tema decisivo é constituído pela formação dos e-tutor (Rivoltella, 2006) que provocou diversas iniciativas sobre o território nacional: o plano de formação For-tutor (disponível na internet: http://www.spazi.org/fortutor/index.php), organizado pelo Ufficio Scolastico della Lombardia (Departamento Escolar da Lombardia), ${ }^{2}$ em colaboração com a Universidade Católica de Milano e com a Universidade de Milano Bicocca; o Master di secondo livello in Metodi e tecniche per l'e-tutor della scuola (disponível na internet: http:/ /www4.unicatt.it/master/MI/E-Tutor/allegati/E-tutor\%20pieg.pdf) organizado por essas duas universidades; o curso de especialização Perfezionamento in Formazione Continua in e-Learning - gestione e tutoring (disponível na internet: http://www.marche.istruzione.it/ comunicati/com_19012007.shtml), organizado por quatro universidades da região de Marche, em colaboração com o Ufficio Scolastico Regionale (Departamento Escolar Regional). ${ }^{3}$ 


\section{Universidade}

A partir do final dos anos de 1990, o e-learnig constituiu um elemento de inovação e desenvolvimento também para o sistema universitário pelo menos em duas direçôes.

A primeira coincide com a criação de estruturas acadêmicas (Centro di Ateneo) dirigidas à pesquisa metodológica, tecnológica e didática e para a implementaçáo de sistemas de e-learning, seja em função da ativação de cursos de graduação a distância, seja para favorecer a integração das tecnologias didáticas aos suportes que os professores usam em aula, segundo modalidades mais tradicionais. A razão desta escolha está mais voltada para a questão de marketing do que para a reflexão sobre a efetiva qualidade da didática. A proliferação das universidades e das sedes universitárias descentralizadas em todo o território italiano, de fato, exigiu que estas mesmas universidades qualificassem a própria oferta para posicionarem-se no mercado da formação e tornarem-se competitivas em relação à concorrência para atrair matrículas: um dos elementos distintivos em relação a isso foi reconhecido no próprio e-learning enquanto fator de modernidade, capacidade de interpretar as exigências da sociedade da informação, elemento de continuidade com o mercado das profissōes. Por outro lado, o aumento da idade média dos estudantes universitários e o fenômeno do retorno para a formação de muitos trabalhadores levaram a pensar em outras modalidades didáticas que não fosse a freqüência de cursos presenciais em aula: também neste caso o e-learning foi reconhecido como escolha funcional.

Este último elemento incidiu sobre a segunda direção de desenvolvimento do e-learning na universidade italiana, ou seja, o nascimento da universidade telemática. Na Itália, não existia uma Open University nacional, como aconteceu na Inglaterra, Alemanha (com a FernUniversität) ou Espanha (com a UNED), visto que politicamente fezse uma escolha oposta, ou seja, a de favorecer uma difusão capilar de sedes acadêmicas em todo o território. Quando, com o duplo impulso do mercado e da necessidade de formação adulta, a exigência se impõe, o Ministero dell'Università e della Ricerca (Ministério da Universidade e da Pesquisa) não opta pela constituição de apenas uma universidade nacional, mas de mais universidades. O Decreto Ministerial de 
17 de abril de 2003 sobre universidades telemáticas sanciona o nascimento delas, alimentando ao mesmo tempo um forte debate no interior da comunidade acadêmica, sobretudo em relação ao fato de que a universidade telemática não poderia garantir os mesmos padrões de qualidade das universidades tradicionais: de fato, dificilmente pode investir em pesquisa e não pode garantir aos estudantes além de lições, faltando completamente nessas universidades a intensa vida cultural, da qual a freqüência universitária sempre permite participar (Ardizzone \& Rivoltella, 2003).

Hoje são 12 as universidade telemáticas italianas regularmente constituídas; ${ }^{4}$ são muito ativas, sobretudo no terceiro nível da formação (mestrado) e na recuperação dos créditos dos estudantes que, por vários motivos, abandonaram os estudos universitários e que, graças ao reconhecimento dos exames já feitos, vêem na universidade telemática uma oportunidade de conseguir o título que não puderam obter antes.

\section{Empresa}

Depois dos entusiasmos do final dos anos de 1990, ligados à ascensão da New Economy, os investimentos ligados ao e-learning precisavam considerar a configuração típica da empresa italiana, caracterizada pela presença de $70 \%$ de pequenas e médias empresas. Isso fez com que só $30 \%$ das grandes empresas (holding, multinacionais) tenham podido dotar-se de um sistema de formação em e-learning, frequentemente parte da atividade da sua Corporate University; para todas as outras empresas, os custos de produção e gestão de e-learning demonstraram ser muito altos e, definitivamente, insustentáveis. O resultado foi a contração dos investimentos e, em certo sentido, a parada do desenvolvimento deste setor de mercado do qual se está saindo recentemente, sobretudo graças às exigências de formação das categorias profissionais.

$\mathrm{Na}$ Itália, os médicos foram os primeiros a adotar uma normativa (ECM, Educazione Continua in Medicina, Educação Continuada em Medicina, disponível na internet: http://www.ministerosalute.it/ecm/ ecm.jsp) que impõe a todos os trabalhadores do setor médico-sanitário (médicos, enfermeiros, técnicos, biólogos, analistas, psicólogos) uma 
capacitação anual obrigatória por meio da participação em cursos de formação. Evidentemente, o e-learning constitui uma ótima oportunidade neste sentido, seja pelo elevado número de trabalhadores do setor (mais de um milhão), seja porque permite aderir à formação sem penalizar a própria presença no lugar de trabalho.

A partir do exemplo das profissões médico-sanitárias, muitos outros profissionais estão fazendo uma normativa análoga: geômetras, consultores financeiros e comerciais, contadores, seguradores. Trata-se de um mercado importante que promete revitalizar todo o setor de $e$ learning nos próximos anos, invertendo finalmente a lógica através da qual a "penetração" do e-learning na empresa foi guiada até agora: de fato não se trata mais de convencer as empresas da utilidade do $e$ learning, mas de responder com e-learning a uma exigência concreta que parece estar satisfeita apenas com a flexibilidade das modalidades formativas típicas do on-line.

\section{Da normalização do e-learning a Web 2.0}

Além dos problemas que evidenciamos no parágrafo precedente, nos últimos dois/três anos o e-learning foi objeto de um claro processo de normalização na Itália. E isso pode ser entendido em dois sentidos. Antes de tudo, pode-se pensar no sentido de que Kuhn (1978) fala da "ciência normal", isto é, da fase do desenvolvimento científico em que um paradigma se impóe e é aceito pela comunidade científica. De outro lado, vale também a referência a Vattimo (1994), quando se lamenta daquela que ele chama "normalização da hermenêutica", isto é, a fase histórica em que é normal para cada um reconhecer-se em uma abordagem hermenêutica a tal ponto que, num contexto no qual todos se dizem hermeneutas, o risco é de que ninguém mais o seja.

Falar de normalização do e-learning significa fazer referência a ambos os processos: o e-learning tornou-se "normal" porque ninguém mais o coloca em discussão, foi integrado nos processos de formação; por outro lado, é difícil encontrar alguém que não preveja um espaço por mais limitado que seja para o e-learning na sua atividade de formação. Trata-se de um resultado que poderia parecer muito positivo e, de certo ponto de vista, o é. O que não é positivo é que a normalização parece estar coincidindo também com uma neutralização daquilo que 
no e-learning é ligado à inovação e à transformação. De fato, a fase normal do e-learning é feita dos mesmos ingredientes padrôes que são possíveis reconhecer em todas as experiências: a adoção de uma plataforma, o esquema "leitura-do-material-discussão-em-fórum", a adoção de um modelo blended (um pouco presencial, um pouco a distância). Os limites desta linha de intervenção são evidentes: a plataforma nem sempre serve, é rígida, requer competências de administração; depois dos primeiros anos em que era levada pela novidade, a produção discursiva no interior de um fórum foi esvaziando-se de sentido, sendo difícil de animar, não incide sobre as aprendizagens; enfim, o blended, superficialmente interpretado como um mix de presença e distância, favorece a reapropriação do e-learning em práticas de aula mais tradicionais (Rivoltella, 2007).

É um impasse do qual provavelmente se poderá sair somente aceitando que uma temporada acabou e que é preciso começar outra. Os prenúncios desta transição já podem ser vistos na afirmação social dos instrumentos da Web 2.0, mas também na consciência de que estas aplicações podem ser usadas pelo "e-learning normal". Pensemos, neste propósito, na difusão dos blogs escolares, na adoção da tecnologia wiki como substituta da intranet empresarial nas pequenas e médias empresas (neste sentido, o software open source T-Wiki está tornandose um verdadeiro caso - conforme consta na internet: http://twiki.org/), mas também na adoção do Google Apps como plataforma de serviço Web por muitos ateneus italianos, entre os quais as Universidades de Messina, Ferrara e Camerino, a exemplo daquilo que já está acontecendo nos Estados Unidos, na Arizona State University, ou na Europa, na Universidade de Linkoping, na Suécia.

A tendência de desenvolvimento é guiada por uma nova dupla representação das tecnologias didáticas que indica a superação do paradigma do e-learning: em primeiro lugar, um "repensar do blended no sentido dos TELE (Technology Enhance Learning Environment): ambientes de aprendizagem não necessariamente telemáticos, ambientes de aprendizagem no sentido amplo do termo, apoiados, potencializados, equipados, integrados pela tecnologia" (Rivoltella, 2007, p. 1086); em segundo lugar, em continuidade com a lógica dos TELE, a reconceitualização dos instrumentos telemáticos das tecnologias da distância às tecnologias de grupo (Parmigiani, 2008), aos instrumentos, 
graças aos quais se favorece a dimensão social da aprendizagem e da inteligência (Coleman, 2007), independentemente da intenção de reduzir o impacto da distância.

\section{Recebido em junho de 2008 e aprovado em julho de 2008.}

\section{Notas}

1. 1998 é o ano a partir do qual a penetração social da internet na Itália torna-se fenômeno de massa, com a intenção de suprir as lacunas de consumo que, antes deste ano, ainda se separava dos padrôes norte-americanos e europeus.

2. No sistema italiano, o Ministério da Instrução Pública articula-se sobre o território no Uffici Scolastici Regionali (USR), Departamento Escolar Regional, e no Uffici Scolastici Provinciali (usP), Departamento Escolar Micro-Regional, segundo a organização administrativa local.

3. No sistema universitário italiano, os Cursos de Especialização, Corsi di Perfezionamento, e o Mestrado, Master, pertencem ao assim chamado terceiro nível de formação. Eles possuem a função de possibilitar ao graduado de primeiro nível (curso de três anos) e de segundo nível (curso de dois anos) competências específicas relacionadas a uma determinada profissão. O mestrado se distingue em master de primeiro nível e de segundo nível, relacionados ao fato de que se dirigem aos graduados trienais ou especialistas (na lógica do processo de Bologna, todo o sistema universitário foi, de fato, reorganizado segundo o esquema $3+2$ ).

4. Trata-se de: Università Telematica "Guglielmo Marconi" (http://www.unimarconi.it/uni/v20/ indexa1.php?id=05124), Università TEL-MA (http://www.unitelma.it/sito/index.php), Università E-Campus (http://www.uniecampus.it/), UniPegaso (http://www.unipegaso.it/ website/home.php), Università "Leonardo da Vinci" (http://www.unidav.it/), Università "Giustino Fortunato" (http://www.unifortunato.eu/), UniSu (http://www.unisugela.it/), Unitel (http://www.uni-tel.it/), Universitas Mercatorum (http://www.unimercatorum.it/), IUL (http://www.iuline.it/), além do já citado consórcio NETTUNO.

\section{Referências}

ARDIZZONE, P.; RIVOLTELLA, P.C. Didattiche per l'e-learning: metodi e strumenti per l'innovazione dell'insegnamento universitario. Roma: Carocci, 2003 (trad. esp: Didactica para e-learning: métodos e instrumentos para la innovacion de la enseñanza universitaria. Malaga: Aljibe, 2004).

BARRETT, E. (Org.). Sociomedia, multimedia, hypermedia. Cambridge, Mass.: MIT, 1992.

CALVANI, A. Rete, comunità e conoscenza: costruire e gestire dinamiche collaborative. Trento: Erickson, 2005. 
CALVANI, A.; ROTTA, M. Comunicazione e apprendimento in Internet: didattica costruttivistica in rete. Trento: Erickson, 1999.

CALVANI, A.; ROTTA, M. Fare formazione in internet. Trento: Erickson, 2000.

COLEMAN, D. Social intelligence. New York: Bantam, 2007.

CRISPIANI, P.; ROSSI, P.G. (Org.). E-learning: formazione, modelli, proposte. Roma: Armando, 2006.

DI NICOLA, P.; COMUNEllo, F. (Org.). Le@rning. Milano: Guerini \& Associati, 2003.

FERRARI, S. Giochi di rete: metodi e strumenti per l'analisi psicopedagogia del forum. Milano: Guerini \& Associati, 2006.

FERRI, P. E-learning: didattica, comunicazione e tecnologie digitali. Firenze: Le Monnier, 2005.

GALLIANI, L.; COSTA, R. (Org.). E-learning nella didattica universitaria: modelli, ricerche ed esperienze della Facoltà di Scienze della Formazione dell'Università di Padova. Napoli: Edizioni Scientifiche Italiane, 2005.

GALLINO, L. La progettazione di ambienti di apprendimento ciberspaziali. In: RiccIARDI, M. (Org.). Scrivere, comunicare, apprendere con le nuove tecnologie. Torino: Bollati Boringhieri, 1995.

GHISLANDI, P. (Org.). E-Learning: didattica e innovazione in università. Trento: Editrice Universitaria, 2002.

KUHN, T.S. A estrutura das revoluções cientificas. São Paulo: Perspectiva, 1978.

LODRINI, T. (Org.). Didattica costruttivista e ipermedia. Milano: Franco Angeli, 2002.

NIPPER, S. Third generation distance learning and computer conferencing. In: Mason, R.D.; KaYE, A. (Ed.). Mindwave: communication, computers and distance education. Oxford: Pergamon, 1989. p. $63-73$. 
NOVARA, D. La diffusione delle teorie e dei metodi di Paulo Freire in Italia. In: Telleri, F. (Org.). Il metodo Paulo Freire: nuove tecnologie e sviluppo sostenibile. Bologna: Clueb, 2002.

PARMIGIANI, D. Tecnolgie di gruppo. Trento: Erickson, 2008.

RANIERI, M. E-learning: modelli e strategie didattiche. Trento: Erickson, 2005.

RIVOLTELLA, P.C. La scuola in rete: temi e problemi di cooperazione on line. Santhià: GS, 1999.

RIVOLTELLA, P.C. Costruttivismo e pragmatica della comunicazione on line: didattica e socialità in internet. Trento: Erickson, 2003.

RIVOLTELLA, P.C. (Org.). E-tutor: profilo, metodi e strumenti. Roma: Carocci, 2006.

RIVOLTELLA, P.C. L'e-learning, tra didattica e ricerca: sfondi concettuali e strategici. In: Rossi, P. (Org.). Progettare l'eLearning/ eLearning design. Macerata: EUM, 2007. p. 1081-1092.

SCURATI, C. (Org.). E-learning/università. Milano: Vita e Pensiero, 2004 .

TRENTIN, G. Telematica e formazione a distanza: il caso Polaris. Milano: Franco Angeli, 1999.

TRENTIN, G. Dalla formazione a distanza all'apprendimento in rete. Milano: Franco Angeli, 2001.

VATTIMO, G. Oltre l'interpretazione. Bari: Laterza, 1994. 